\title{
Apolipoprotein CIII may mediate the impacts of angiopoietin-like protein 8 on triglyceride metabolism
}

\author{
Mengdie Luo, Xin Su, Yuhong Yi, Yang Yang and Daoquan Peng ${ }^{*}$
}

\begin{abstract}
Background: Angiopoietin-like protein 8(ANGPTL8) and apolipoprotein CIII (apoCIII) were found to inhibit the activity of lipoprotein lipase ( $L P L)$ and disrupt the clearance of triglyceride-rich lipoproteins (TRLs), leading to hypertriglyceridemia. Whether any relationship exists between these two important modulators of triglyceride metabolism has not been reported. Besides, whether ANGPTL8 concentration is altered in the patients with coronary artery disease (CAD) is still unclear.

Methods: A hospital-based case-control study was conducted. Sixty-eight CAD subjects and fifty-two nonCAD controls were recruited. Plasma apoCIII, ANGPTL8 was measured.

Results: ANGPTL8 and apoCIII concentration exhibited no significant difference between CAD group and nonCAD group. Both ANGPTL8 and apoCIII were significantly correlated with triglyceride level $(r=-0.243, P=0.008 ; r=0.335$, $P<0.001$, respectively). Regression analysis revealed that apoCIII was an independent contributor to triglyceride level independent of ANGPTL8 concentration (standardized $\beta=0.230, P<0.01$ ).
\end{abstract}

Conclusion: ApoCIII may mediate the effects of ANGPTL8 on triglyceride metabolism.

Keywords: ANGPTL8, apoCIII, Triglyceride, Coronary artery disease

\section{Background}

The angiopoietin-like proteins (ANGPTL1-8) are secreted glycoproteins sharing common structure but exerting distinct physiological effects [1]. ANGPTL8, also referred to as betatrophin, lipasin, refeeding-induced in fat and liver (RIFL) and hepatocellular carcinoma-associated protein (TD26), was found to be a novel player in lipid metabolism [2]. Recent findings revealed that ANGPTL8, together with ANGPTL3 and ANGPTL4, controlled by nutritional status, could regulate triglyceride metabolism by inhibiting the activity of lipoprotein lipase (LPL) [3, 4], the rate-limiting enzyme for triglyceride hydrolysis and plasma triglyceride clearance [5]. Different researches have shown that ANGPTL8 concentrations were altered in diseases such as obesity, diabetes mellitus, metabolic syndrome [2] and non-alcoholic fatty liver disease (NAFLD) [6]. However,

\footnotetext{
* Correspondence: pengdq@csu.edu.cn

Department of Cardiovascular Medicine, the Second Xiangya Hospital,

Central South University, Changsha 410011, Hunan, China
}

whether ANGPTL8 concentration is altered in the patients with coronary artery disease (CAD) has not been reported.

Liver-derived apolipoprotein CIII (apoCIII), mainly presents on the surface of triglyceride-rich lipoproteins (TRLs) and high-density lipoprotein (HDL) [7], was proved to be a crucial regulator of triglyceride metabolism [8-10]. ApoCIII could inhibit the binding of TRLs with LPL and impair TRLs clearance [7], which deteriorated lipid disorders and accelerated CAD progression. Although ANGPTL8 and apoCIII exerted similar effects on triglyceride metabolism, the relationship between these two triglyceride modulators was still unclear.

In the present study, we conducted an observational study to examine the ANGPTL8 and apoCIII level in different groups classified by the anthropometric and metabolic profiles. Besides, the relationship between ANGPTL8 and apoCIII and their effects on triglyceride metabolism were also studied.

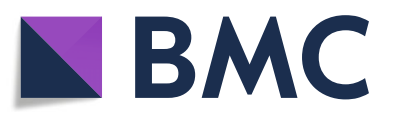

(c) The Author(s). 2018 Open Access This article is distributed under the terms of the Creative Commons Attribution 4.0 International License (http://creativecommons.org/licenses/by/4.0/), which permits unrestricted use, distribution, and reproduction in any medium, provided you give appropriate credit to the original author(s) and the source, provide a link to the Creative Commons license, and indicate if changes were made. The Creative Commons Public Domain Dedication waiver (http://creativecommons.org/publicdomain/zero/1.0/) applies to the data made available in this article, unless otherwise stated. 


\section{Methods}

\section{Subjects}

We recruited 68 CAD subjects and 52 nonCAD subjects from the Department of Cardiovascular Medicine of the Second Xiangya Hospital, Central South University. In this study, CAD patients were mainly composed of acute coronary syndrome (ACS), including ST-segment elevated myocardial infarction (STEMI), non ST-segment elevated myocardial infarction (NSTEMI) and unstable angina. ACS was diagnosed by the clinical symptoms and signs, ischemic electrocardiographic abnormalities, and coronary angiography showing $\geq 50 \%$ stenosis in at least one main coronary artery. The exclusion criteria included: a history of renal failure, chronic hepatic diseases, high fever, or bacterial/viral infection, autoimmune disease, arthritis, malignancies, severe diabetes and hypertension, and other severe medical illnesses.

\section{Clinical and biochemical measurements}

Patient information, including age, gender, smoking and drinking history, and statin therapy history, was recorded. The details of anthropometric measurements (weight, height, body mass index) were assessed after overnight fasting for at least $10 \mathrm{~h}$. Peripheral blood samples were obtained from patients' brachial veins. Subjects fasted for at least $10 \mathrm{~h}$ before blood collection and then blood routine, urine routine, concentrations of lipid parameters, including total cholesterol (TC), triglyceride (TG), low-density lipoprotein cholesterol (LDL-C), HDL-C, apoAI, apoB, free fatty acid (FFA), were evaluated via an automated analyzer (Hitachi P7600). Concentrations of high-sensitivity C-reactive protein (hsCRP) were measured with a latex particle, enhanced immunoturbidimetric assay. For the subsequent experiments, fresh plasma was obtained by centrifugation at $3000 \mathrm{r} / \mathrm{min}$ at $4{ }^{\circ} \mathrm{C}$ for $10 \mathrm{~min}$. The plasma was aliquoted and stored at $-80{ }^{\circ} \mathrm{C}$ freezer until analysis.

\section{Measurement of plasma apoCIII and ANGPTL8}

Plasma ApoCIII and ANGPTL8 concentration were measured with commercially available ELISA kits (apoCIII: Abcam, ab154131, UK; ANGPTL8: EIAAB, E11644H, Wuhan, China). All the measurement of plasma apoCIII and ANGPTL8 were performed in duplicate for each sample. The coefficient of variation for intra- and inter-assay variation was $<6$ and $<9 \%$, respectively.

\section{ApoB-depleted plasma preparation}

According to previous reported procedures [11], 540ul heparin sodium solution ( $280 \mathrm{mg} / \mathrm{ml}$, Aladdin, H104201) and $10 \mathrm{ml}$ manganese chloride solution $(1.06 \mathrm{~mol} / \mathrm{L}$, Aladdin, M112542) were mixed. 100ul mixed solution was added to $1 \mathrm{ml}$ plasma, incubated for $30 \mathrm{~min}$ at $4{ }^{\circ} \mathrm{C}$, followed by centrifugation at $1500 \mathrm{~g}$ for $30 \mathrm{~min}$. Supernatant was collected. If supernatant was still turbid (especially samples from patients with hypertriglyceridemia), plasma was centrifuged at $12000 \mathrm{~g}$ for $10 \mathrm{~min}$ again. Previous study revealed that heparin sodium/manganese chloride precipitation had no effects on HDL size as well as cholesterol efflux measurement [12], and therefore this method was chosen to prepare apoB-depleted plasma in the study.

\section{Statistical analysis}

Statistical analysis was performed with Statistical Package for Social Sciences version 22.0 and plots were made with GraphPad Prism V.6.0 (GraphPad Software, Inc., La Jolla, California, USA). Clinical data are expressed as mean \pm standard deviation (normally distributed continuous data) or median with interquartile range (skewed distributed continuous data). Comparisons between categorical data were performed with Chi Squared tests, while continuous variables were assessed by unpaired $t$ test (for normal distribution) or nonparametric test (for skewed distribution). For the variables skewed distributed, logarithmatic-transformed values were used for the analysis. To evaluate the associations between variables, partial correlation analysis was performed. A two tailed $P$ value $<0.05$ was considered statistically significant.

\section{Results}

Characteristics of subjects

Demographic and biochemical characteristics of participants are shown in Table 1. The study includes 120 unrelated individuals, $60.80 \%$ of the participants were male and the mean age was 64.17 years. Compared to nonCAD controls, CAD subjects had higher free fatty acid (FFA). Besides, the percentage of diabetic subjects and statin users was also significantly higher in CAD group.

\section{ANGPTL8 and ApoCIII concentrations in subjects}

ApoCIII and ANGPTL8 concentrations were measured in CAD group and nonCAD group (Table 2). Although the differences between these two groups were not significant, the apoCIII $\mathrm{HDL}_{\mathrm{L}}$ concentration and apoCIII $\mathrm{HDL}$ ratio was significantly higher in CAD group than that in nonCAD group (Table 2). In addition, subjects were classified according to their apoCIII and ANGPTL8 concentrations (Table 3). Subjects were divided according to their plasma apoCIII level: low apoCIII group (plasma apoCIII < median $11.7 \mathrm{mg} / \mathrm{dl}$ ) and high apoCIII group (plasma apoCIII $\geq$ median $11.7 \mathrm{mg} / \mathrm{dl}$ ). Subjects in the low apoCIII group exhibited significantly higher ANGPTL8 than those in the high apoCIII group [569.6(432.2-917.6) vs $447.8(339.8-827.1), P=0.036$, Fig. 1a]. Triglyceride level in the low apoCIII group was significantly lower than 
Table 1 Anthropometric and metabolic characterisitcs of study participants

\begin{tabular}{|c|c|c|c|c|}
\hline Variables & All $(n=120)$ & $\mathrm{CAD}(n=68)$ & Non- $C A D(n=52)$ & $P$ value \\
\hline Male(\%) & 60.80 & 60.29 & 61.54 & NS \\
\hline Age(years) & $64.17 \pm 8.11$ & $65.13 \pm 8.03$ & $63.91 \pm 8.06$ & NS \\
\hline $\mathrm{BMI}\left(\mathrm{kg} / \mathrm{m}^{2}\right)$ & $24.43 \pm 3.81$ & $24.49 \pm 4.57$ & $24.47 \pm 3.21$ & NS \\
\hline $\mathrm{TG}(\mathrm{mg} / \mathrm{dL})$ & 117.8(87.7-174.5) & 120.9(91.5-168.1) & 113.4(87.7-182.5) & NS \\
\hline $\mathrm{TC}(\mathrm{mg} / \mathrm{dL})$ & 155.1(133.0-184.8) & 146.6(128.5-183.3) & $160.9(133.0-181.0)$ & NS \\
\hline $\mathrm{HDL}-\mathrm{C}(\mathrm{mmol} / \mathrm{L})$ & $1.09 \pm 0.25$ & $1.05 \pm 0.25$ & $1.12 \pm 0.26$ & NS \\
\hline LDL-C(mmol/L) & $2.54 \pm 0.80$ & $2.40 \pm 0.74$ & $2.48 \pm 0.82$ & NS \\
\hline $\operatorname{ApoAl}(g / L)$ & $1.13 \pm 0.20$ & $1.11 \pm 0.19$ & $1.16 \pm 0.20$ & NS \\
\hline ApoB(g/L) & $0.92 \pm 0.26$ & $0.89 \pm 0.26$ & $0.88 \pm 0.27$ & NS \\
\hline FFA (mmol/L) & $0.48 \pm 0.28$ & $0.51 \pm 0.29$ & $0.42 \pm 0.26$ & 0.026 \\
\hline $\mathrm{hsCRP}(\mathrm{mg} / \mathrm{L})$ & $2.73(1.00-8.98)$ & $2.64(0.71-10.46)$ & $2.30(0.87-4.64)$ & NS \\
\hline $\mathrm{BUN}(\mathrm{mmol} / \mathrm{L})$ & $5.91(4.84-7.23)$ & $5.70(4.35-7.30)$ & $6.28(5.32-7.96)$ & NS \\
\hline UA(umol/L) & $308.1(280.0-405.5)$ & $305.8(283.3-370.9)$ & $341.0(292.1-430.1)$ & 0.054 \\
\hline CR(umol/L) & $74.50(57.20-86.30)$ & 77.15(67.68-84.68) & 77.90(58.98-98.40) & NS \\
\hline Diabetes(\%) & 21.67 & 32.35 & 7.69 & 0.001 \\
\hline Statin use(\%) & 36.67 & 54.41 & 13.46 & $<0.0001$ \\
\hline
\end{tabular}

Values are expressed as mean \pm SD or median (interquartile range). CAD indicates coronary artery disease; $B M I$ body mass index, $T G$ triglyceride, $T C$ total cholesterol, $H D L-C$ high density lipoprotein-cholesterol; $L D L-C$ low density lipoprotein-cholesterol; apoAl apolipoprotein Al, apoB apolipoprotein $B, F F A$ free fatty acid, $h S C R P$ high sensitivity $C$ reactive protein, $B U N$ blood urea nitrogen, UA uric acid, $C R$ creatinine, apoCIII apolipoprotein CIII

that in the high apoCIII group $[113.4(88.1-145.3)$ vs 148.8(114.7-230.7), $P=0.002$, Fig. 1b].

\section{Correlation analysis of clinical variables with ANGPTL8 and apoCIII}

To investigate variables associated with ANGPTL8 and apoCIII, correlation analysis was performed. Correlation coefficients between clinical variables and plasma ANGPTL8 as well as apoCIII concentration (both log-transformed) were present in Table 4. The results showed that ANGPTL8 was positively correlated with age ( $r=0.240, P=0.008)$, but inversely correlated with triglyceride $(r=-0.243, \mathrm{P}=0.008$, Fig. $2 \mathrm{a}$ ), and renal function biomarkers including BUN $(r=0.351, P<0.0001)$, UA

Table 2 Comparison of circulating ANGPTL8 and apoCIII concentrations in CAD group and nonCAD group

\begin{tabular}{|c|c|c|c|c|}
\hline & $\begin{array}{l}\text { All } \\
(n=120)\end{array}$ & $\begin{array}{l}\text { CAD } \\
(n=68)\end{array}$ & $\begin{array}{l}\text { Non-CAD } \\
(n=52)\end{array}$ & $P$ value \\
\hline $\begin{array}{l}\text { ANGPTL8 } \\
(\mathrm{pg} / \mathrm{ml})\end{array}$ & $\begin{array}{l}561.1 \\
(390.1-882.9)\end{array}$ & $\begin{array}{l}544.4 \\
(374.3-903.7)\end{array}$ & $\begin{array}{l}597.7 \\
(408.6-853.1)\end{array}$ & NS \\
\hline $\begin{array}{l}\text { ApoCIII } \\
(\mathrm{mg} / \mathrm{dl})\end{array}$ & $\begin{array}{l}11.7 \\
(9.1-13.5)\end{array}$ & $\begin{array}{l}10.6 \\
(8.8-12.3)\end{array}$ & $\begin{array}{l}11.3 \\
(9.0-14.7)\end{array}$ & NS \\
\hline $\begin{array}{l}\text { apoCl| } \|_{H D L} \\
(\mathrm{mg} / \mathrm{dl})\end{array}$ & $\begin{array}{l}4.55 \\
(3.32-6.68)\end{array}$ & $\begin{array}{l}5.01 \\
(3.81-7.44)\end{array}$ & $\begin{array}{l}3.86 \\
(3.07-6.15)\end{array}$ & 0.048 \\
\hline $\begin{array}{l}\text { apoCIII } \\
\text { ratio }\end{array}$ & $\begin{array}{l}0.43 \\
(0.30-0.53)\end{array}$ & $\begin{array}{l}0.48 \\
(0.31-0.55)\end{array}$ & $\begin{array}{l}0.37 \\
(0.24-0.47)\end{array}$ & 0.003 \\
\hline
\end{tabular}

Data are expressed as mean \pm SD or median (interquartile range). ANGPTL8 indicates angiopoietin-like protein 8; apoCIII, apolipoprotein CIII; apoCIII $\mathrm{HDL}$, apolipoprotein $\mathrm{CIII}$ in apoB-depleted plasma; apoCIII $\mathrm{HDL}_{\mathrm{L}}$ ratio, apolipoprotein CIII in apoB-depleted plasma over plasma apolipoprotein CIII; CAD, coronary artery disease $(r=0.333, P<0.001)$ and CR $(r=0.509, P<0.0001)$. On the other hand, plasma apoCIII presented a positive relationship with triglyceride $(r=0.335, P<0.001$, Fig. $2 \mathrm{~b})$. Besides, ANGPTL8 was inversely correlated with plasma apoCIII ( $r=-0.302, P=0.002$, Fig. 2c).

\section{Multivariate analysis for the associations of clinical variables to triglyceride}

In order to determine the independent contributors to triglyceride, stepwise multiple regression models were fitted after adjustment for different variables (Table 5). Log-transformed values were used for the variables skewed distributed, including ANGPTL8 and apoCIII. In addition, plasma apoCIII level was an independent

Table 3 Comparison of circulating ANGPTL8 and apoCIII concentrations under different baseline conditions

\begin{tabular}{llll}
\hline & $\begin{array}{l}\text { Low apoCIII } \\
(n=65)\end{array}$ & $\begin{array}{l}\text { High apoCIII } \\
(n=55)\end{array}$ & P value \\
\hline ApoCIII $(\mathrm{mg} / \mathrm{dl})$ & $/$ & $/$ & $/$ \\
apoCIII $_{\text {HDL }}(\mathrm{mg} / \mathrm{dl})$ & $3.9(3.1-5.1)$ & $5.0(3.4-8.2)$ & 0.014 \\
apoCIII $_{\text {HDL }}$ ratio & $0.44(0.36-0.53)$ & $0.34(0.23-0.51)$ & 0.052 \\
ANGPTL8(pg/ml) & $569.6(432.2-917.6)$ & $447.8(339.8-827.1)$ & 0.036 \\
Triglyceride $(\mathrm{mg} / \mathrm{dl})$ & $113.4(88.1-145.3)$ & $148.8(114.7-230.7)$ & 0.002 \\
CAD percentage & $66.2 \%$ & $43.2 \%$ & 0.037 \\
\hline
\end{tabular}

Data are expressed as mean \pm SD or median (interquartile range). ANGPTL8 indicates angiopoietin-like protein 8; apoCIII, apolipoprotein Clll; apoClll HDL,

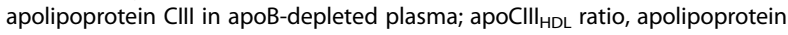
CIII in apoB-depleted plasma over plasma apolipoprotein CIII; CAD, coronary artery disease 


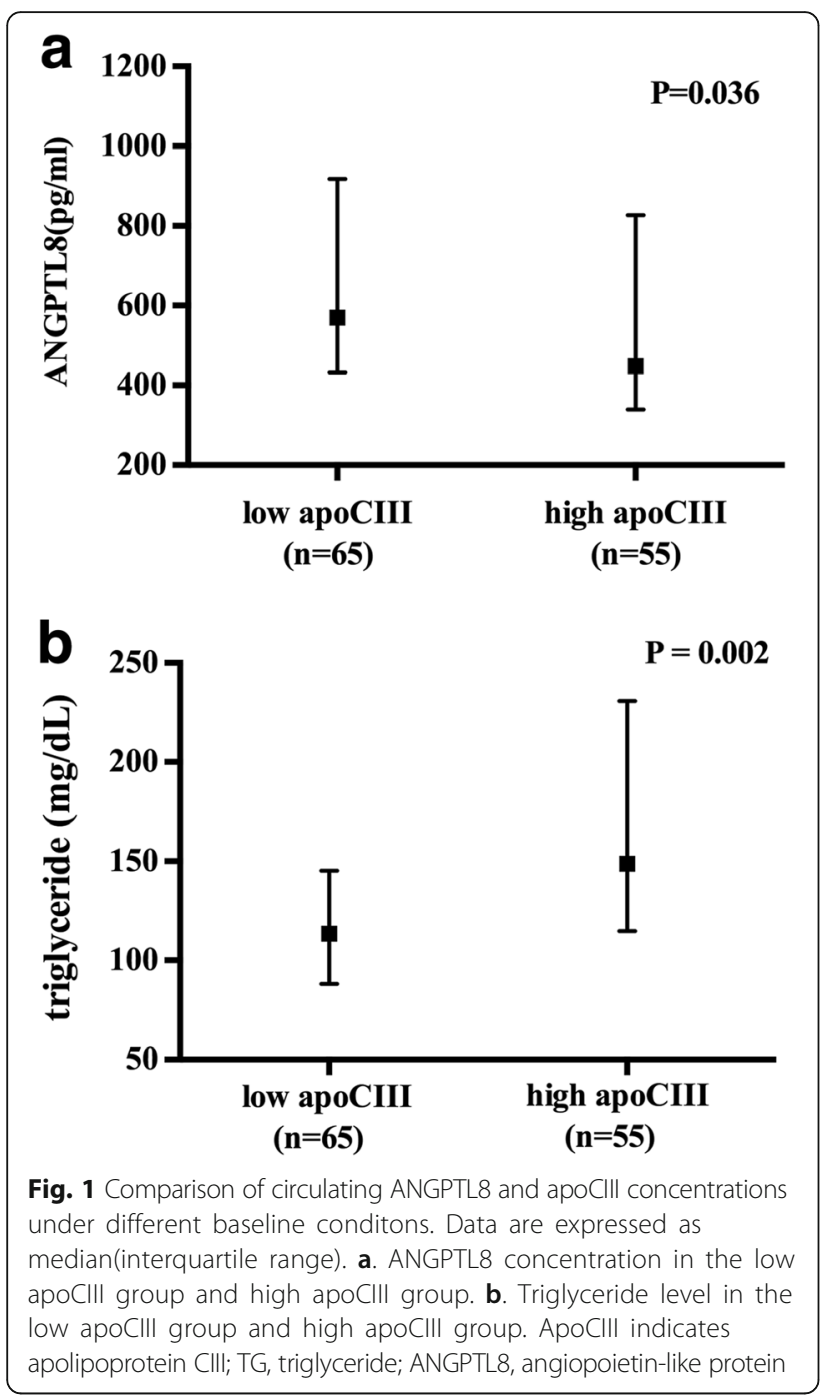

contributor to the triglyceride level. When apoCIII was introduced into the regression model (model 3), the relationship between ANGPTL8 and triglyceride level disappeared.

\section{Discussion}

In this study, we found that ANGPTL8 and apoCIII were significantly correlated with triglyceride level. Besides, stepwise multiple regression analysis revealed that apoCIII was an independent contributor to triglyceride level.

Many clinical studies and animal experiments have shown that ANGPTL8 was highly involved in triglyceride metabolism. ANGPTL8 knockout mice presented nearly $70 \%$ reduced plasma triglyceride levels compared to the wild-type controls after feeding [13], while ANGPTL8 overexpression significantly increased plasma triglyceride levels by five folds [14]. ANGPTL8 was found to inhibit LPL activity and disrupt triglyceride clearance partly via
ANGPTL3 activation [4]. In the cohort of Beijing children and adolescents metabolic syndrome (BCAMS) study, participants with high TG (defined as $\geq 150 \mathrm{mg} / \mathrm{dl}$ ) exhibited significantly increased ANGPTL8 concentration [15]. However, our experiments showed that ANGPTL8 was significantly higher in the low TG group when subjects were divided according to their TG levels. Another study that aimed at dyslipidemic middle-aged cohorts in Caucasian population also found that subjects with lower TG displayed significantly higher ANGPTL8 levels than subjects with higher TG (defined as $\geq 150 \mathrm{mg} / \mathrm{dl}$ ) [16]. The disparity among these population studies might be caused by the sample selection. Young population $(20.2 \pm 2.9$ years old) with risk for metabolic syndrome was selected in BCAMS study [15], while older patients were recruited in the current study $(64.17 \pm 8.11$ years old $)$ and the study aimed at Caucasian middle-aged population study [16]. Besides, ANGPTL8 was also positively correlated with age, and therefore the relationship between triglyceride and ANGPTL8 might be confounded by age.

The physiological regulation of LPL activity is driven via post-translational mechanisms including ANGPTLs (ANGPTL 3, 4 and 8) and apolipoproteins (apoCIII and apoAV) [5]. In circulation, apoCIII, mainly residing on the surface of HDL and TRLs, inhibited LPL activity and disrupted TRLs clearance [7], thereby leading to hypertriglyceridemia. In this study, plasma apoCIII exhibited a relatively strong correlation with triglyceride. Besides, the relationship between ANGPTL8 and triglyceride disappeared when apoCIII was introduced into the regression model (model 3), suggesting that the effect of ANGPTL8 on triglyceride metabolism might be apoCIII dependent. Previous study found that ANGPTL8 regulated LPL activity and triglyceride metabolism partly dependent on ANGPTL3, but other factors beyond ANGPTL3 might also mediate the effects of ANGPTL8 on triglyceride regulation [4]. Our research provided a hint that apoCIII might be involved in ANGPTL8 modulation of LPL activity and triglyceride metabolism. However, whether the interaction exists awaits further investigation.

Interestingly, ANGPTL8 was strongly correlated with biomarkers of renal function (BUN, UA and CR). Although the relationship between ANGPTL8 and eGFR was still controversial $[17,18]$, a recent study conducted on T2DM patients revealed that ANGPTL8 was associated with urinary albumin excretion and renal function [18]. In addition, the study also showed that ANGPTL8 could increase the risk of diabetic nephropathy (DN) and might serve as a predictor for DN progression [18]. Kidney may be the important organ for ANGPTL8 degradation and excretion. When ANGPTL8 could not be efficiently cleaned, accumulated ANGPTL8 might cause dysregulated lipids metabolism in the kidney, leading to lipids accumulation in the artery wall, foam cells formation, 
Table 4 Pearson's correlations between clinical variables and log-transformed ANGPTL8 as well as log-transformed apoCIII in all the subjects

\begin{tabular}{|c|c|c|c|c|c|c|c|c|}
\hline & ANGPTL & & Plasma & & apoCll| & & apoCIII & \\
\hline & r & $P$ value & r & $P$ value & $r$ & $P$ value & $r$ & $P$ value \\
\hline Age & 0.240 & 0.008 & -0.075 & 0.456 & 0.102 & 0.270 & 0.042 & 0.677 \\
\hline BMl & 0.075 & 0.417 & -0.033 & 0.740 & -0.025 & 0.783 & -0.061 & 0.542 \\
\hline CR(log-transformed) & 0.509 & $<0.0001$ & 0.012 & 0.909 & 0.120 & 0.196 & 0.096 & 0.342 \\
\hline BUN(log-transformed) & 0.351 & $<0.0001$ & -0.107 & 0.291 & 0.064 & 0.494 & 0.050 & 0.623 \\
\hline UA(log-transformed) & 0.333 & $<0.001$ & 0.131 & 0.194 & 0.179 & 0.053 & -0.035 & 0.731 \\
\hline TG(log-transformed) & -0.243 & 0.008 & 0.335 & $<0.001$ & -0.241 & 0.008 & -0.316 & 0.001 \\
\hline TC(log-transformed) & -0.245 & 0.007 & 0.198 & 0.046 & -0.102 & 0.270 & -0.012 & 0.904 \\
\hline HDL-C & -0.002 & 0.975 & -0.059 & 0.553 & 0.135 & 0.144 & 0.302 & 0.002 \\
\hline LDL-C & -0.224 & 0.014 & 0.165 & 0.097 & -0.069 & 0.453 & -0.020 & 0.845 \\
\hline apoAl & -0.102 & 0.270 & 0.108 & 0.283 & -0.014 & 0.877 & 0.117 & 0.245 \\
\hline apoB & -0.167 & 0.069 & 0.208 & 0.037 & -0.104 & 0.263 & -0.064 & 0.522 \\
\hline hsCRP(log-transformed) & 0.067 & 0.471 & 0.067 & 0.505 & 0.131 & 0.156 & -0.013 & 0.901 \\
\hline ANGPTL8(log-transformed) & / & / & -0.302 & 0.002 & 0.089 & 0.331 & 0.158 & 0.113 \\
\hline ApoCIII(log-transformed) & -0.302 & 0.002 & / & / & 0.319 & 0.001 & -0.217 & 0.029 \\
\hline apoCIII $\left.\right|_{H D L}($ log-transformed) & 0.089 & 0.331 & & & / & / & 0.796 & $<0.0001$ \\
\hline apoCIII ${ }_{\text {HDL }}$ ratio & 0.158 & 0.113 & & & 0.796 & $<0.0001$ & / & / \\
\hline
\end{tabular}

$B M I$ indicates body mass index, $C R$ creatinine, $B U N$ blood urea nitrogen UA uric acid, TG triglyceride, $T C$ total cholesterol, $H D L-C$ high density lipoprotein-cholesterol, $L D L-C$, low density lipoprotein-cholesterol, apoAl apolipoprotein $\mathrm{Al}$, apoB apolipoprotein $\mathrm{B}, h s C R P$ high-sensitivity $\mathrm{C}$ reactive protein, $A N G P T L 8$ angiopoietin-like protein 8 , apoCIII apolipoprotein CIII, apoCIII ${ }_{H D L}$ apolipoprotein CIII in apoB-depleted plasma, apoCIII ${ }_{H D L}$ ratio apolipoprotein CIII in apoB-depleted plasma over plasma apolipoprotein CIII
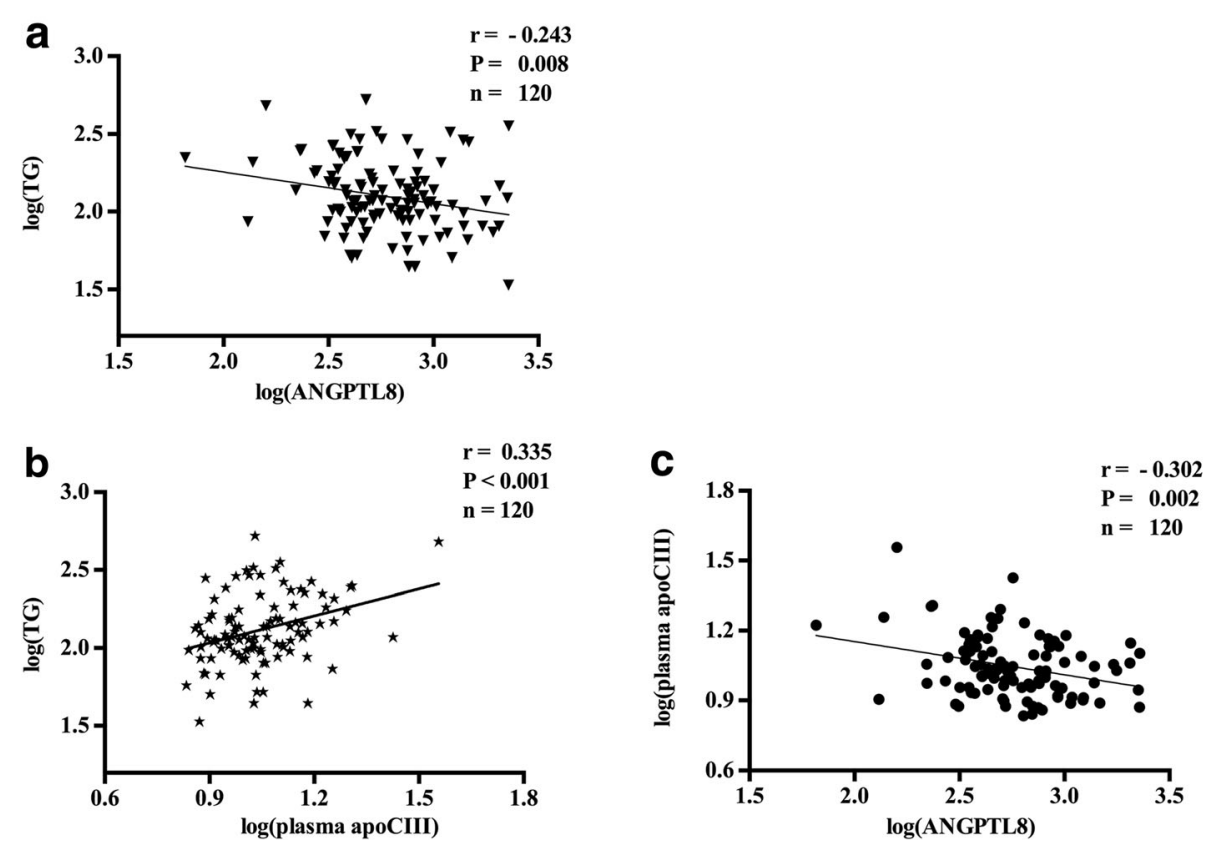

Fig. 2 Pearson's correlations between clinical variables and log-transformed ANGPTL8 as well as log-transformed apoCIII in all the subjects. a. ANGPTL8 and TG. b. ApoCIII and TG. c. ANGPTL8 and apoCIII. TG indicates triglyceride; ANGPTL8, angiopoietin-like protein; apoCIII, apolipoprotein CIII 
Table 5 Independent contributors to the triglyceride level

\begin{tabular}{|c|c|c|c|c|}
\hline & R square & $\beta$ & Standardized $\beta$ & $P$ value \\
\hline Model 1 & 0.207 & & & $<0.001$ \\
\hline Age & & -0.005 & -0.171 & 0.056 \\
\hline $\mathrm{HDL}-\mathrm{C}$ & & -0.269 & -0.308 & $<0.001$ \\
\hline ANGPTL8 (log-transformed) & & -0.170 & -0.203 & 0.020 \\
\hline Model 2 & 0.234 & & & $<0.001$ \\
\hline Age & & -0.005 & -0.189 & 0.028 \\
\hline $\mathrm{HDL}-\mathrm{C}$ & & -0.265 & -0.303 & $<0.001$ \\
\hline ApoCIII (log-transformed) & & 0.153 & 0.259 & 0.002 \\
\hline Model 3 & 0.257 & & & $<0.001$ \\
\hline Age & & -0.004 & -0.153 & 0.079 \\
\hline $\mathrm{HDL}-\mathrm{C}$ & & -0.273 & -0.312 & $<0.001$ \\
\hline ANGPTL8 (log-transformed) & & -0.133 & -0.159 & 0.064 \\
\hline ApoCIII (log-transformed) & & 0.136 & 0.230 & 0.006 \\
\hline
\end{tabular}

HDL-C indicates high density lipoprotein-cholesterol, ANGPTL8 angiopoietin-like protein 8; apoCIII apolipoprotein CIII

atherosclerosis deterioration and glomerulosclerosis occurrence [19].

We acknowledged the limitations of our study. First, the cross-sectional nature of our study did not provide the direct proof for the causality. Additionally, we only measured ANGPTL8 in the fasting state, but ANGPTL8 activity was regulated by nutritional status [14]. Therefore the association of ANGPTL8 and apoCIII in the postprandial state still needed to be validated in the future research. Finally, we measured ANGPTL8 with EIAAB ELISA kits that recognizes N-terminus and measures the full-length form of ANGPTL8. Although our research presented similar ANGPTL8 levels with previous studies adopting the same kind of ELISA kit, the adoption of different ELISA kits which measured different forms of ANGPTL8 might cause some discrepancies in the results [20].

\section{Conclusions}

In conclusion, our results showed that circulating ANGPTL8 presented a strong relationship with biomarkers of renal function, including BUN, UA and CR. Moreover, ANGPTL8 was inversely correlated with plasma apoCIII and the association between ANGPTL8 and triglyceride disappeared when plasma apoCIII was taken into consideration, suggesting the potential role of plasma apoCIII in ANGPTL8 action. Further research is warranted to elucidate the relationship between apoCIII and ANGPTL8 and the underlying mechanism of triglyceride regulation.

\section{Abbreviations}

ANGPTL8: Angiopoietin-like protein 8; apoCIII: apolipoprotein CIII; CAD: coronary artery disease; DN: diabetic nephropathy; HDL: high-density lipoprotein;

LDL-C: low-density lipoprotein cholesterol; LPL: lipoprotein lipase;

NAFLD: non-alcoholic fatty liver disease; RIFL: refeeding-induced in fat and liver; TRLs: triglyceride-rich lipoproteins

\section{Funding}

This project was supported by grants from National Natural Science Foundation of China (No.81670426).

\section{Availability of data and materials}

All data generated or analyzed during this study are included in this manuscript.

\section{Authors' contributions}

Conception and design: DP and ML; Experiments performance: ML, XS and YYi; Analysis and interpretation of the data: ML and DP; Article drafting and revision: $M L, D P$ and YYang. All authors reviewed the manuscript. All authors read and approved the final manuscript.

Ethics approval and consent to participate

All the subjects provided written informed consent. The study was approved by the Medical Ethics Committee of the Second Xiangya Hospital of Central South University and was conducted in accordance with approved guidelines and regulations.

\section{Competing interests}

The authors declare that they have no competing interests.

\section{Publisher's Note}

Springer Nature remains neutral with regard to jurisdictional claims in published maps and institutional affiliations.

Received: 2 September 2017 Accepted: 15 May 2018

Published online: 18 July 2018

References

1. Dijk W, Kersten S. Regulation of lipid metabolism by angiopoietin-like proteins. Curr Opin Lipidol. 2016;27:249-56.

2. Zhang $R$, Abou-Samra AB. A dual role of lipasin (betatrophin) in lipid metabolism and glucose homeostasis: consensus and controversy. Cardiovasc Diabetol. 2014;13:133.

3. Fu Z, Abou-Samra AB, Zhang R. A lipasin/Angptl8 monoclonal antibody lowers mouse serum triglycerides involving increased postprandial activity of the cardiac lipoprotein lipase. Sci Rep. 2015;5:18502.

4. Quagliarini F, Wang Y, Kozlitina J, Grishin NV, Hyde R, Boerwinkle E, Valenzuela DM, Murphy AJ, Cohen JC, Hobbs HH. Atypical angiopoietin-like protein that regulates ANGPTL3. Proc Natl Acad Sci U S A. 2012;109:19751-6.

5. Kersten S. Physiological regulation of lipoprotein lipase. Biochim Biophys Acta. 2014:1841:919-33.

6. Lee YH, Lee SG, Lee CJ, Kim SH, Song YM, Yoon MR, Jeon BH, Lee JH, Lee BW, Kang ES, et al. Association between betatrophin/ANGPTL8 and non-alcoholic fatty liver disease: animal and human studies. Sci Rep. 2016:6:24013.

7. Norata GD, Tsimikas S, Pirillo A, Catapano AL. Apolipoprotein C-III: from pathophysiology to pharmacology. Trends Pharmacol Sci. 2015;36:675-87.

8. Jorgensen AB, Frikke-Schmidt R, Nordestgaard BG, Tybjaerg-Hansen A. Loss-of-function mutations in APOC3 and risk of ischemic vascular disease. N Engl J Med. 2014:371:32-41.

9. Tg, Hdl Working Group of the Exome Sequencing Project NHL, blood I, Crosby J, Peloso GM, Auer PL, Crosslin DR, Stitziel NO, Lange LA, Lu Y, et al: loss-of-function mutations in $\mathrm{APOC}$, triglycerides, and coronary disease. $\mathrm{N}$ Engl J Med 2014, 371:22-31.

10. Olivieri O, Bassi A, Stranieri C, Trabetti E, Martinelli N, Pizzolo F, Girelli D, Friso S, Pignatti PF, Corrocher R. Apolipoprotein C-III, metabolic syndrome, and risk of coronary artery disease. J Lipid Res. 2003;44:2374-81.

11. Warnick GR, Albers JJ. A comprehensive evaluation of the heparin-manganese precipitation procedure for estimating high density lipoprotein cholesterol. J Lipid Res. 1978;19:65-76.

12. Davidson WS, Heink A, Sexmith H, Melchior JT, Gordon SM, Kuklenyik Z, Woollett L, Barr JR, Jones JI, Toth CA, Shah AS. The effects of apolipoprotein B depletion on HDL subspecies composition and function. J Lipid Res. 2016; 57:674-86.

13. Wang Y, Quagliarini F, Gusarova V, Gromada J, Valenzuela DM, Cohen JC, Hobbs HH. Mice lacking ANGPTL8 (Betatrophin) manifest disrupted triglyceride metabolism without impaired glucose homeostasis. Proc Natl Acad Sci U S A. 2013;110:16109-14. 
14. Zhang R. Lipasin, a novel nutritionally-regulated liver-enriched factor that regulates serum triglyceride levels. Biochem Biophys Res Commun. 2012; 424:786-92.

15. Fu J, Hou C, Li L, Feng D, Li G, Li M, Li C, Gao S, Li M. Vitamin D modifies the associations between circulating betatrophin and cardiometabolic risk factors among youths at risk for metabolic syndrome. Cardiovasc Diabetol. 2016;15:142.

16. Gomez-Ambrosi J, Pascual-Corrales E, Catalan V, Rodriguez A, Ramirez B, Romero S, Vila N, Ibanez P, Margall MA, Silva C, et al. Altered concentrations in dyslipidemia evidence a role for ANGPTL8/Betatrophin in lipid metabolism in humans. J Clin Endocrinol Metab. 2016;101:3803-11.

17. Ebert T, Kralisch S, Hoffmann A, Bachmann A, Lossner U, Kratzsch J, Bluher M, Stumvoll M, Tonjes A, Fasshauer M. Circulating angiopoietin-like protein 8 is independently associated with fasting plasma glucose and type 2 diabetes mellitus. J Clin Endocrinol Metab. 2014:99:E2510-7.

18. Chen CC, Susanto H, Chuang WH, Liu TY, Wang CH. Higher serum betatrophin level in type 2 diabetes subjects is associated with urinary albumin excretion and renal function. Cardiovasc Diabetol. 2016;15:3.

19. Vaziri ND. HDL abnormalities in nephrotic syndrome and chronic kidney disease. Nat Rev Nephrol. 2016;12:37-47.

20. Fu Z, Abou-Samra AB, Zhang R. An explanation for recent discrepancies in levels of human circulating betatrophin. Diabetologia. 2014;57:2232-4.

Ready to submit your research? Choose BMC and benefit from:

- fast, convenient online submission

- thorough peer review by experienced researchers in your field

- rapid publication on acceptance

- support for research data, including large and complex data types

- gold Open Access which fosters wider collaboration and increased citations

- maximum visibility for your research: over $100 \mathrm{M}$ website views per year

At BMC, research is always in progress.

Learn more biomedcentral.com/submissions 